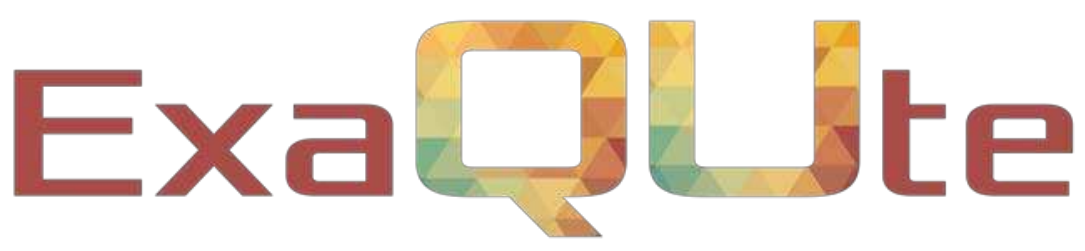

Exascale Quantification of Uncertainties for

Technology and Science Simulation

\title{
D8.3 Report on exploitation activities
}

\section{Document information table}

\begin{tabular}{|l|l|}
\hline Contract number: & 800898 \\
\hline Project acronym: & ExaQUte \\
\hline Project Coordinator: & CIMNE \\
\hline Document Responsible Partner: & TUM \\
\hline Deliverable Type: & Report \\
\hline Dissemination Level: & PUblic \\
\hline Related WP \& Task: & WP 8 Task 8.2 \\
\hline Status: & Final version \\
\hline
\end{tabular}

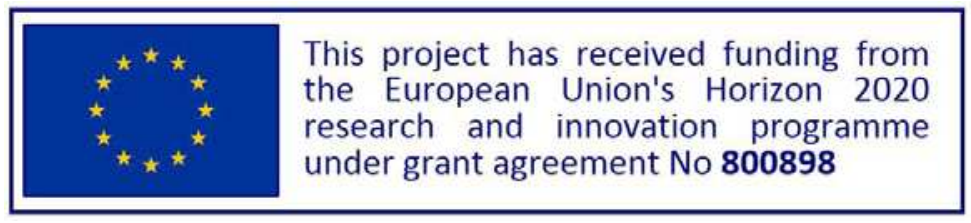




\section{Authoring}

\begin{tabular}{|l|l|l|l|l|}
\hline Prepared by: & Partner & Modifications & Version & Comments \\
\hline Authors & CIMNE & create & & \\
\hline Cecilia Soriano & TUM & Compile inputs & & \\
\hline Andreas Apostolatos & & & & \\
\hline & & & & \\
\hline Contributors & CIMNE & & & \\
\hline Cecilia Soriano & CIMNE & & & \\
\hline Riccardo Rossi & & & & \\
\hline & & & & \\
\end{tabular}

\section{Change Log}

\begin{tabular}{|l|l|l|} 
Versions & Modifications & Comments \\
\hline 1 & Sections of the report & First version
\end{tabular}

2. Input from partners 2nd

3- Final Edition

Approval
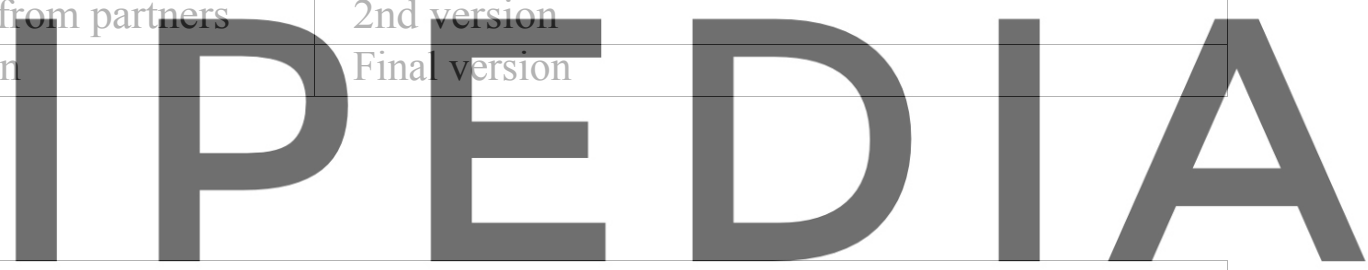

Approved by:

Register for free at https/ywww.scipedia.com to Partwhiload the version without the Watermark Task leader Cecilia Sorlano

\begin{tabular}{|l|l|l|l|l|}
\hline WP leader & Andreas Apostolatos & TUM & $29 / 11 / 2019$ & OK \\
\hline Coordinator & Riccardo Rossi & CIMNE & $29 / 11 / 2019$ & OK \\
\hline
\end{tabular}




\section{Table of contents}

1 Aim of the exploitation Plan in ExaQUte .................................................................4

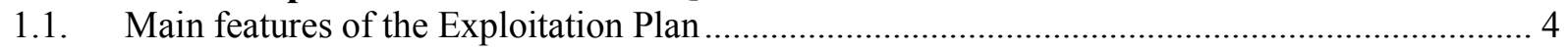

1.2. Strategy for knowledge management and protection: .......................................................... 5

2 Exploitation plans of the partners ..................................................................................6

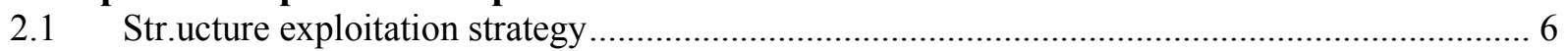

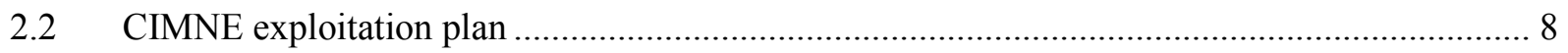

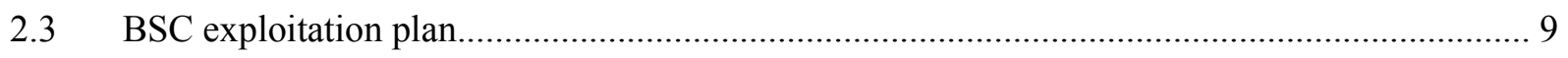

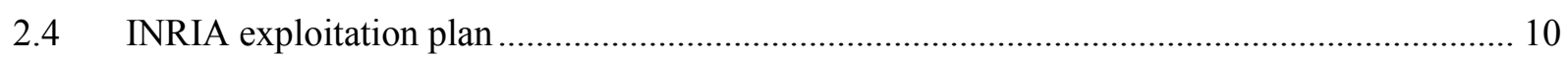

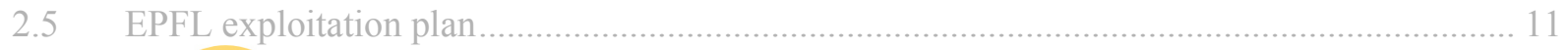

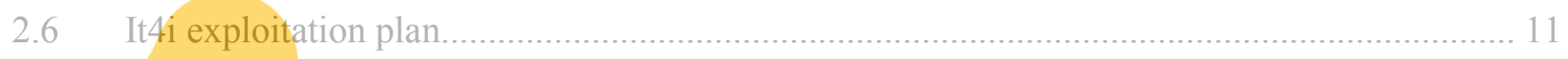

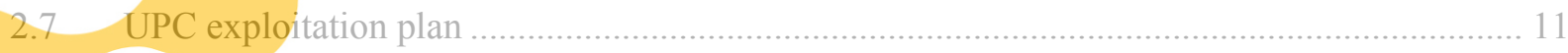

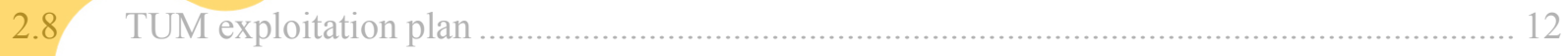
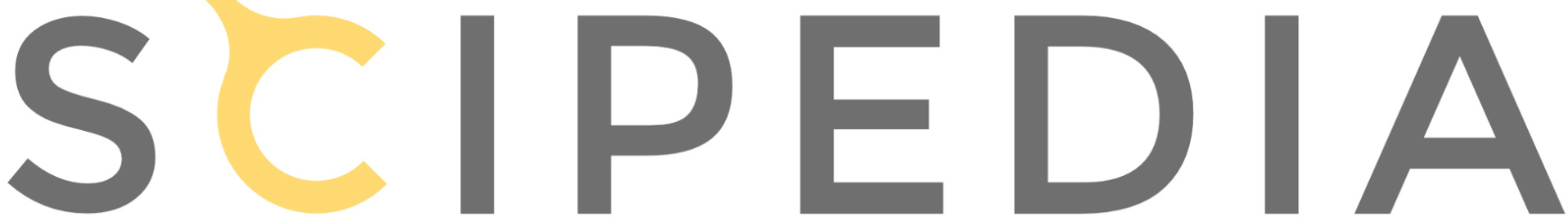

Register for free at https//www.scipedia.com to download the version without the watermark 


\section{Aim of the exploitation Plan in ExaQUte}

The process of exploiting the results of the project is directed towards optimizing its value, strengthening its impact, improving its transference and integrating it in a sustainable way and using them actively in systems and practices at local, regional, national and European levels.

Nowadays, every research center should have a vocation for transferring the scientific and technical outputs from RTD projects to the industrial sector. This is effectively carried out in cooperation with companies from different sectors that exploit and market the RTD's technology. In the case of CIMNE, it has actively promoted the creation of spinoff companies, some of them totally or partially owned, that play an important role in the industrialization of CIMNE technology. In that framework, the company CIMNE TECNOLOGIA SA (www.cimnetecnologia.com ) was created in November 2011 by CIMNE to protect the intellectual property rights, industrialize and market the technology and prototypes developed at CIMNE on a national and international level. This kind of initiatives from the partners are be used to foster the transference into the market of the results of the ExaQUte project.

Some particularities of this project imply that most of its developments will be generated and shared as a common technology more than making it privative. In fact, most of the scientific results of ExaQUte are, or will be, made available in the form of an open-source solver which incorporate the contributions from the different project partners (in line with the KRATOS philosophy explained before in this proposal). Such parts will be developed on top of the partner's open-source background and will remain open, so to foster future research as well as the future participation of the research and engineering community at large.

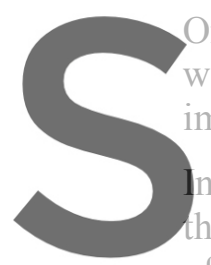

On the other hand, purely te

within a Graphical User implementations, which

other words, the key idea

market and the foundati

of a code typically relies in its usability
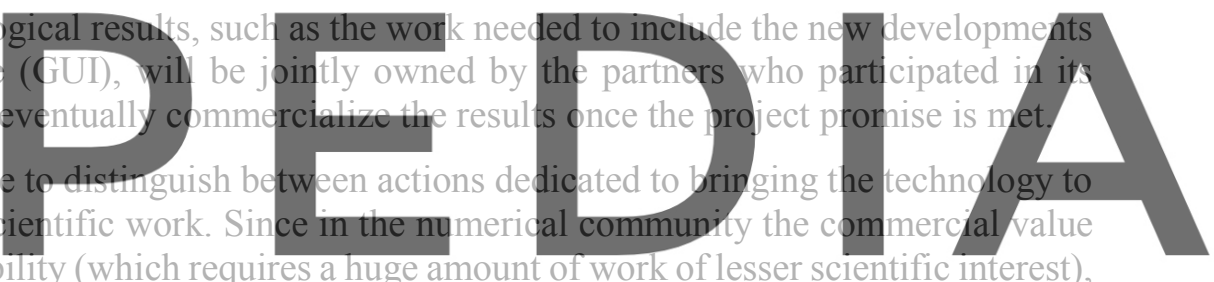

the strategy of keeping the solver module open-source and to eventually commercialize the user-interface

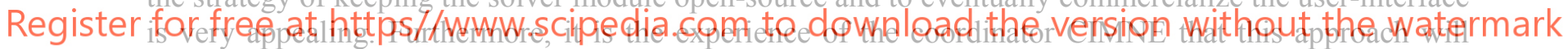

guarantee the smooth collaboration with the project commercial partner, while providing a guarantee to

the scientific actors not to be restricted in future research.

\subsection{Main features of the Exploitation Plan}

The main outcomes of ExaQUte that could be exploited (either economically or as knowledge acquisition) individually or/and collectively by the partners are:

- Knowledge and understanding about advanced numerical methods for HPC. It will offer a competitive advantage compared to other research centers.

- This knowledge could be easily transferred to different areas of engineering. The exploitation strategy will take into consideration the proprietary rights of the partners, and the protection of the foreground. It will not contradict any issue regarding the use of existing background.

- The newly-obtained simulation tools with high parallelization and scalability capabilities.

Regarding the possible exploitation of this last result, the partners are aware that, as of today, the possibility to run on HPC platforms is mostly restricted to leading research centers and administration, and to some large industries. However, it is expected that in the future access to these types of infrastructures, or at least to multi-core platforms that will benefit from the highly parallelized codes, will be much more extended, In fact some FP7 and H2020 running initiatives under the umbrella of the I4SME (such as projects FORTISSIMO, CloudSME, or CloudFlow) are working towards this end. Even some high-end industries 
will be able to own (more modest) computing infrastructures to run large-scale applications on real engineering problems.

Different strategies of exploitation are envisioned, based on previous experience of the partners with the codes and tools developed prior to this project. These different approaches can be summarized as:

- Open source availability of the developed software to ensure highest impact and enrichment from the interactions with users with whom to exchange knowledge and experiences. This is the approach that has been followed by CIMNE with its KRATOS Platform (http://www.cimne.com/kratos/). Following the successful KRATOS philosophy, ExaQUte could use similar tools to ensure best spreading and tuning of the outputs of the project, ensuring its extension and continuity after the end of the project.

- Free access to the compiled code.

- Availability of parts of the tools and libraries developed during the project.

- Commercial exploitation of the developed tools through specific licensed industrial applications, which make use of the tools developed in the project but integrate them in a user-friendly tool to cover the specific needs of a particular sector

To initiate our exploitation activities, we will perform a survey of potential users and vendors (in particular SMEs) of applications related to our solvers. Given the extent and distribution of that community, we might then focus on direct marketing activities to directly reach end customers or attempt to exclusively or nonexclusively sub-license our solutions to vendors who would include them into their already established application suites. Naturally, we will also evaluate the possibility of compiling a stand-alone, multifunctional software package to be sold as an individual produet by the consortium's commercial partnei. This, however, would : updates.

1.2. Strategy for
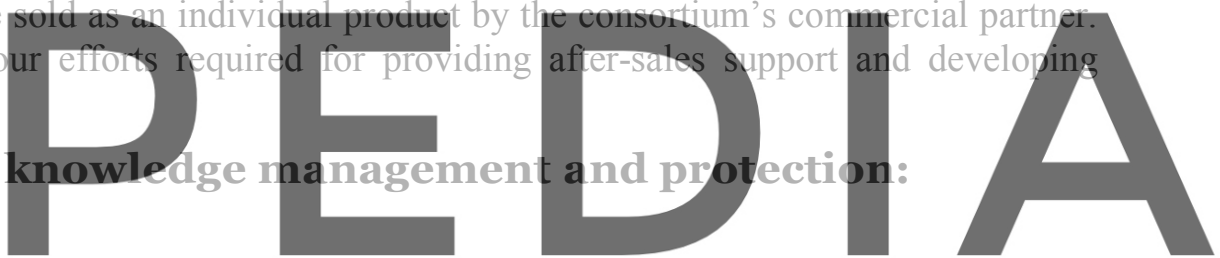

Regarding IPR, and following EC recommendations, the partners made use of the DESCA 2020 model

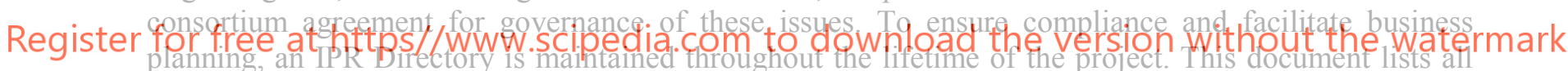

items of knowledge relating to the work of the project (both pre-existing know-how and results developed in the project), and make explicit for each item: the owner(s); the nature of the knowledge, and its perceived potential for exploitation; the currently agreed status of the item concerning access rights, plans to use the knowledge in; exploitation, or plans to disseminate it outside the Consortium; measures required, or in place, to ensure protection of IPR for the item.

All members have been granted access rights to background needed for carrying out the project on a royalty-free basis. Access rights to background needed to use the foreground must also be granted by the members on a royalty-free basis.

In order to settle details of the co-operation such as license policies, granting of rights and patenting the Consortium will enter into an agreement (called the "Transaction") regarding the ownership of foreground and access rights to be provided to any SME and the remuneration paid to it by a RTD performer. This transaction corresponds to part of Annex I of the EC Grant Agreement and any modification thereof has to be subject to approval of the Commission. The intellectual property rights and licensing regulations follows the recommendations of the Commission. However, the following basic principles concerning IPR and exploitation rights are agreed upon that:

- Background of all partners is granted royalty free to the partners during the course of the project.

- The use of any party's background information for the purpose of the project's results will be on fair, reasonable and favorable conditions.

- Partners manage access to the necessary know-how by other partners and impulse and manage any possible additional patent on project result. 
The following measures are taken to secure IPR in the published reports:

- The exploitation manager will play a key role in the screening and publication of project results. His tasks will include the co-ordination and monitoring of the publication of results, taking into account the need to protect information. All publications must be approved by the exploitation manager;

- Partners involved in producing the results concerned must agree to publication of the data and details;

- Publications will be identified as the results of a European RTD project;

\section{Exploitation plans of the partners}

The consortium of ExaQUte is composed of 8 partners. 7 of them are Universities or Research centers and 1 is an SME (Str.ucture Gmbh).

It is clear that mission, objectives and goals are different for non-profit and for-profit entities, but still all of them can have planes regarding the transfer of outcomes resulting of their research work.

This section describes possible scenarios that have been identified for exploiting the results of ExaQUte.

There will be in general two different types of results for exploitation the ExaQUte outputs. The one type of result is the know-how and experiences gathered within the project, while the other type are the specific software products. The first can be used e.g. within consultancy, while the second one can be sold directly to customers as a product

In the next subsection the partners oals and its capabilities

2.1 Str.ucture exploitation strategy
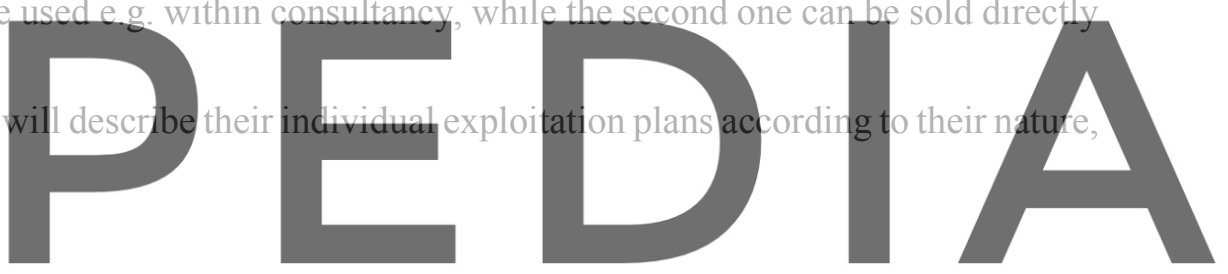

Register for free at https//www.scipedia.com to download the version without the watermark

In the proposal the SME participating in ExaQUte (Str.ucture GmbH) anticipated the following exploitation plan describing the impact that the project will have in their business. At the time of writing this deliverable, its content is still applicable and for the time being no changes have been foreseen.

One of Str.ucture's main engineering activities is the industrial application of advanced computer-aided simulation methods on parallel HPC platforms for the investigation of wind-loaded building structures. The most important projects which have been designed and built are the world's largest convertible shading systems for the Great Mosques in Makkah and Madina, Saudi Arabia with more than $150000 \mathrm{~m}^{2}$ shaded area.

Str.ucture envisions a great potential applying advanced computational wind engineering methods to the field of high-rise structures, as there is an increasing need worldwide to build more and slender (and hence wind prone) high-rise structures, in densely urbanized parts of the world. Based on str.ucture's experience on the wind simulation of large-scale flexible light-weight structures, the new field of application is targeted. A basic setup is already ongoing by collaborating with the company Maurer AG (http://www.maurer.eu/), one of the world leading manufacturing companies for vibration control systems (like Tuned Mass Dampers). First studies of wind induced vibrations on a $435 \mathrm{~m}$ high-rise structure located in Russia, have already been performed.

Another market str.ucture is aiming for, is offering special engineering and consultancy services for the investigation of bridge aerodynamics. Long-span cable stayed and suspension bridges are built world-wide with project costs ranging from $100 \mathrm{Mio} €$ to 2 Bio $€$ and respective design costs. Str.ucture is collaborating with the special engineering office Leonhard Andrä \& Partners (http://www.lap-consult.com/), a worldleading office in this field. Str.ucture is targeting to extend its engineering portfolio offering computational wind engineering services. 
Str.ucture expect to take advantage of the technical developments of the of the project results as follows:

- The embedded methods with its parallel mesh generation and adaptive refinement: They allow the computational investigation of arbitrary and complex geometrical shapes. A common trend of world's leading architectural design offices like Zaha Hadid Architects (http://www.zahahadid.com), Gerkan Mark \& Partners (http://www.gmp-architekten.de/projekte.html) and many others are designing complex lightweight building shapes which are not able to be analyzed by standard procedures from building codes. Standard wind tunnel testing procedures are extremely costly or unfeasible due to the difficulties in correctly reproducing complex structures in small scale model.

- The technological innovation of Space-Time Methods and Dynamic Scheduling will overcome one of the main drawbacks that currently appear in wind engineering problems for building structures: the time needed for the computation of millions of time steps to cover a large amount of wind storms for satisfactory statistical safety.

- Technological develonments regarding uncertainty quantifications are required to predict statistically safe peak values of reaction forces, displacements and accelerations on which structural design is based. As each building structure is a prototype by itself whose performance is not tested during its lifetime in mockups, robust and reliable design procedures are essential to achieve the required level of safety according to building standard.

Str.ucture expect to take economic advantage of the developments of the of the project results as follows:

- Available commercial software is not specifically designed for civil-engineering applications. Thus,

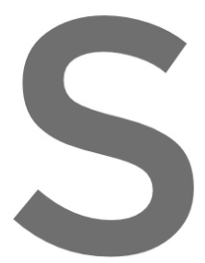

it could not be optin

(UQ, OUU'). Furthert

the processor count

example, for a typicitin

license can cost abou
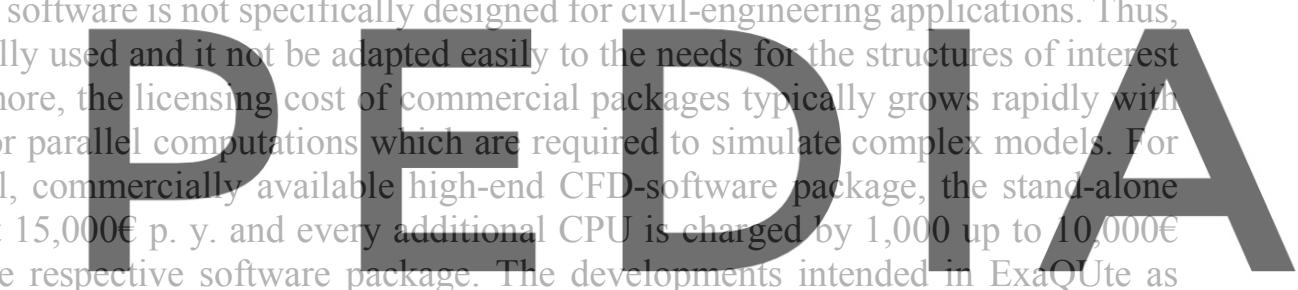

Open-Source software packages will overcome this problem.

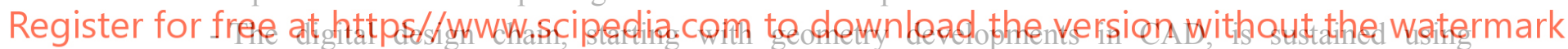

computational methods. Compared to the traditional approach using physical testing, this allows in turns an easy use of computational simulation tools in all phases of building projects. The information content will increase which will lead to reduced costs. Furthermore, the future extension of simulation capabilities to thermal comfort, fire safety and building physics problems is feasible. As a result, the efficiency of the design \& analysis iterations is increased drastically. This is a unique selling point in the portfolio of str.ucture compared to its competitors.

- The development of the proposed simulation software, tailored to the specific needs of building structures, will allow reducing costs by increasing the information content in the first design phases using simulation results. The digital design chain, starting with geometry developments in CAD, is sustained using computational methods solving engineering work packages especially on wind load assessments, thermal comfort, fire safety and building physics problems

- The increase of information content in the initial planning phases by means of fast-to-run variant studies avoid costs due to changes in late planning phases. The most effective technical solution can be achieved with significantly reduced the costs of planning, manufacturing and assembly (Fig. 2.1).

Further advantages of using computational design tools for wind load assessment are an increased analysis efficiency by the use of parametric design tools, avoiding extensive experimental testing by using the computational design environment

It is expected that the ExaQUte project will lead to a technological impulse of str.ucture: The fields of activity of str.ucture and also the number of potential clients are increased. It is expected that this will result in an increase of turnover of $15 \%$ in 2018 and $25 \%$ in 2019 and $25 \%$ in 2020. 
Pioneering the know-how and application of advanced digital simulation tools in the construction sector will lead to str.ucture's leadership in this field. Innovative building solutions could be offered. Thus, development and validation of a virtual wind tunnel are in the center of strategic and commercial interests of the company since it constitutes the basis of ongoing competitiveness and innovation leadership in this field.
Design development of building structures

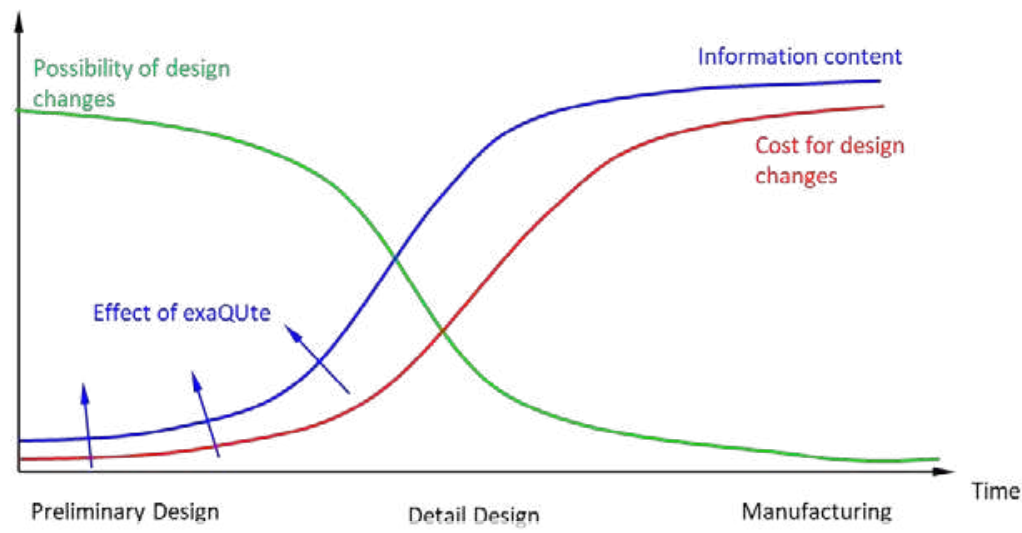

Design development cycle for building structures. Effect of ExaQUte

\subsection{CIMNE exploitation plan}

The implementation of ExaQUte mainly results in a collection of methodologies, algorithms and codes that will improve the competitiveness of CIMNE in the area of extreme-scale simulation of scientific and engineering problems.

We list below the main 1 the main benefits that the $\mathrm{C}$

ExaQUte has facilitated the development of too open-source solver plat form $\mathrm{K}$ ditcones result
an obtain from the
RATOS (www.c ted from the project
is that have impro
imne.com/l $\mathbf{r a t o s}$. types of products (of different nature regarding its exploitations vision) are been generated:

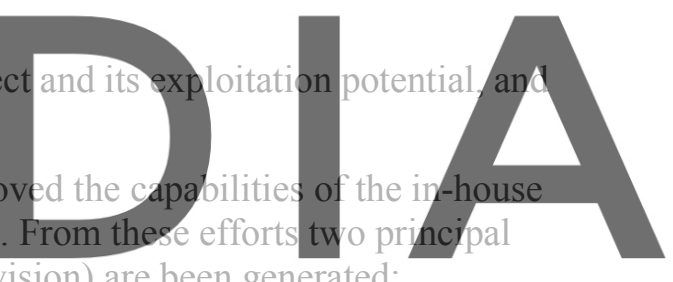

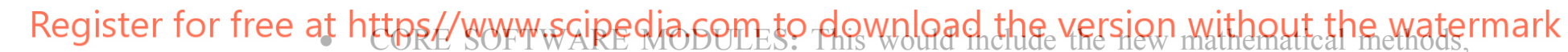
algorithms and solvers for large-scale for multiphysics problems that enhanced the capabilities, robustiness and efficiency of KRATOS.

As an open-source platform, these software modules will be freely accessible by the computational community worldwide, thereby reinforcing the position of CIMNE as a leader regarding open-source simulation codes for solving large-scale problems in engineering and applied sciences, which invariably will open many opportunities for new cooperative projects with universities, research centers and industries in the five continents.

- APPLICATIONS: A collection of applications have been implemented in ExaQUte that have been useful to test the new software developments under large-scale real word scenarios. The more relevant applications have to do with the interest of the SME participating in the consortium and has to do with the simulation and optimizations of wind-loaded building structures:

ExaQUte is helping to reinforce the position of CIMNE in the path of HPC research, and confirms the capability of CIMNE to design, coordinate and lead ambitious research project in this field. Being an institution whose lion's share of its budget comes from competitive sources, ExaQUte success has increased the reputation of the center in the HPC community, and has positioned CIMNE in the leading group in the "fight" for funding resources, increasing our chances to be funded in more projects.

Some examples of this positive feedback have to do with the possibility to participate in the calls of the European EuroHPC High-Performance Computing Joint Undertaking, where we will be submitting several projects. 
Similarly, the existence of ExaQUte has allowed CIMNE to participate in competitive calls that are open only to entities awarded with a FET project.

The FET Innovation Launchpad call aims at turning results from FET-funded projects into genuine societal or economic innovations. These projects are focused on the non-scientific aspects and the early stages of turning a result of an ongoing or recently finished FET project into an innovation with socio-economic impacts.

Together with the colleagues at BSC participating in ExaQUte, last October 2019 we submitted a proposal to this call whose aims to develop a software tool (builder) to produce Digital Twins that run on the Edge (this means that they are installed and operate on the physical asset they represent, enabling a new breed of novel real-time applications, from autonomous vehicles to small devices.). The project would democratize the use of digital twins extending its application to market segments and users (i.e. SMEs) currently without access this emerging technology. In addition, this software tool will be open source, deploying and freely sharing source code as needed. This goes in line with the approach followed by successful spin offs which also take advantage of community driven projects.

Finally, ExaQUte is also helping to train young scientists that will become highly-skilled researchers in the field of HPC, ensuring the continuation in the long run of the research carried out in a field that is strategic for CIMNE.

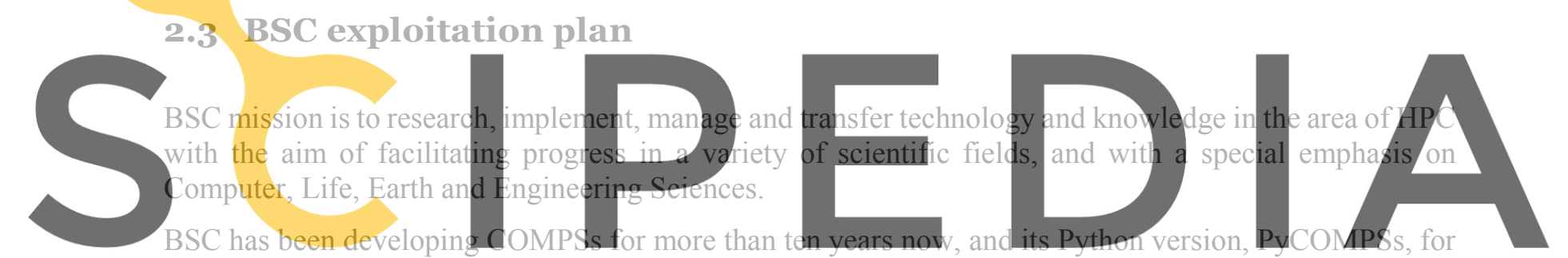

the last five years. PyCOMPSs is a task-based programming model that enables the parallel execution on

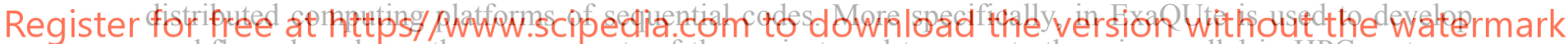
workflows based on other components of the project, and to execute them in parallel in HPC systems. PyCOMPSs/COMPSs offers an asynchronous parallel execution mode, alternative to the more traditional MPI parallel programming model, suited for the type of applications (MLMC) to be developed in the project.

In ExaQUte, BSC is further extending PyCOMPSs to support multiple functionalities:

- Task failure management mechanism: PyCOMPSs will be able to perform different management of tasks' failures according to hints given by the programmer at development time. For example, if a task fails, the programmer may have indicated to the runtime to ignore the failure and continue with the execution, to cancel the successors of the task in the workflow or to perform the default behavior, by safely finishing the whole workflow. This feature enables that large stochastic runs are not finished when a single individual task fails.

- Tasks' groups, exceptions and tasks cancellation: programmer can now define a group of tasks. Also, tasks can through exceptions that are captured by the main program of the workflow. In the occurrence of an exception, the runtime will cancel the ongoing task groups but the program can define the behavior to implement from that point. This will be applied in ExaQUte in some speculative patterns of the MLC algorithm.

- Optimization for locality and storage with MPI tasks': the tasks in PyCOMPSs can be MPI tasks, i.e., tasks that are implemented with this parallel programming model and involve for their execution multiple nodes. In ExaQUte, the workflows will involve MPI tasks that may be using different number of processes and data can be then used between the different MPI tasks with different access patterns. For this reason, it is important to enable PyCOMPSs with a way of 
indicating this access patterns, and to implement policies in the runtime that optimizes the data transfers, to exploit the locality of the data and also to reduce the amount of stored data.

BSC is exploiting PyCOMPSs in research projects and collaborations as a programming, execution and data management framework for parallel applications in fog to cloud and HPC environments.

Additionally, PyCOMPSs is used in production in the BSC supercomputers and in the Spanish Supercomputing Network as a programming model for workflows and applications not following the more traditional MPI model. The extensions developed during the project are published in the releases that are performed every 6 months and made available as open source for the community.

Additionally, as well as contributing to the state of the art with research papers in relevant conferences and journals, PyCOMPSs helps to improve and update courses and seminars given as part of BSC training, and courses taught by BSC staff at Universitat Politècnica de Catalunya.

Finally, as mentioned in the section above, CIMNE and BSC have submitted a proposal to the H2020FETlaunchpad call to explore the exploitation of a software tool (builder) to produce Digital Twins that run on the Edge"

\subsection{INRIA exploitation plan}

The Mmg platform (www.mmgtools.org) is an open source platform distributed under the L-GPL

license. This license has been chosen to ensure to the community access to Mmg, and to allow at the same time the exploitatio

Since few years, due to somentis (https://github.com/MmgT

annual event to meet users community has grown. This gathers academics and industrial partners interested

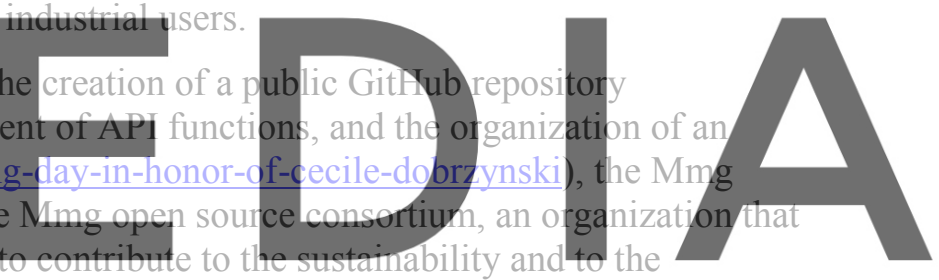
development of the Mmg tools. The financial support of this partners allows the hiring of a full-time

The aim of Inria within the ExaQUte project is to develop a library on top of the Mmg remesher to perform parallel mesh adaptation (the ParMing library): indeed, due to the difficulty to parallelize remeshers, they remain serial while solvers have been parallelized more and more efficiently. Today, with the easy access to larger and larger cluster, it is not anymore possible to ask to the solver developers to merge the mesh on one rank in order to perform serial mesh adaptation during a parallel simulation: the adapted mesh become too expensive in term of memory. This is why, with the work done in ExaQUte, we hope:

- That we will satisfy the increasing number of Mmg users asking for a parallel remesher;

- That we will provide to the community one of the first L-GPL parallel remesher, which will help to keep ahead of other remeshers;

- That we will target new users that can't perform mesh adaptation because they handle meshes that can't be processed by a serial tool;

- That this parallel library will contribute to convince more users to become members of the Mmg consortium.

As side effects to the collaborations involved in the ExaQUte project:

- The post-doctoral researcher hired for the library development is a new potential user and contributor of both Mmg and ParMmg (and will contribute to the platform promotion and development);

- A new CIMNE researcher has been trained to the ParMmg interfacing; 
- The ParMmg library will be a basis for future research work on parallel mesh adaptation (loadbalancing, interface migration, efficient way to handle dependencies...).

\subsection{EPFL exploitation plan}

The ExaQUte project already lead to the development and release of a Python library for hierarchical Monte Carlo methods in HPC environments. Its flexibility and novel capabilities make it a useful tool for testing and developing algorithms, which will be valuable in future research projects.

Other scientific tools will be developed in the next stages of the project-e.g. computational tools for optimization under uncertainty. Although they will be adapted to the specific needs of the final application in civil engineering targeted in ExaQUte, they are relevant in many other areas of computational science and engineering (e.g. aerospace, finance) and will be exploited in other ongoing or future research projects at EPFL.

Along with these concrete productions, this research effort also helps to further the expertise at EPFL in fields of importance in both science and industry, such as optimization under uncertainties.

More generally, ExaQUte is an invaluable opportunity to train young researchers on international projects, and foster synergetic collaboration between European research teams. Beyond the benefits for the scientists directly involved in it, ExaQUte provides a wealth of topics and results that we plan to exploit in scientific courses and student projects at EPFL

These immaterial exploitations will be formalized and made visible in open research publications whenever possible.

\subsection{It4i exploitation plan}

As a supercomputing center, IT4I frequently organizes workshops and tutorials for both academic and industry users of its supercomputers. The ExaQUte project, and its results and potential will be presented during these workshops, and tutorial sessions will be used to teach users how to use the project outcomes for increasing the exascale potential of methods for numerical modeling and simulations.

As part of the VSB-Technical University of Ostrava, students of the MSc programme focused on HPC as well and other subjects related to Computational Fluid Dynamics will receive proper training in using developer tools.

Sincw addressing uncertainties during CFD simulations is another key factor in increasing fidelity of the reasults, participation in the ExaQUte project provides IT4I with a unique opportunity to broaden its capabilities and use this knowledge in the future research projects.

IT4I as a Digital Innovation Hub will present ExaQUte project and its outcomes to the industrial companies in the Czech Republic and through its network of DIHs Europewide as well. Tutorials and workshops will be organized for potential users of ExaQUte technologies. Developed tools could be offered to the industrial partners through Solver-as-a-service scheme. Capabilities to solve complex problems will be exploited in future projects of collaborative and contracted research.

\subsection{UPC exploitation plan}

As a public institution dedicated to higher education and research, within the fields of architecture, engineering and technology, the mission of UPC is to create knowledge and develop technology but also to make this technology available to the society. With this mission in mind, exploitation of research is an important aspect of its activities. 
Within UPC, the group of numerical analysis and scientific computing, AniComp, aims to develop, analyse, implement and apply numerical methods in engineering and applied sciences. Although its primary concern is scientific production (see deliverable 8.2 in this respect), it also contributes to the development of FEMPAR, described in the project proposal.

The exploitation plan at UPC is based on providing FEMPAR through a free and open source software license. This model permits the collaborative work within the same platform of researchers in the field of scientific computing, exploiting the unique scalability properties of the FEMPAR code (we note, once again, that FEMPAR was included in the High-Q club of the most scalable scientific codes in Europe). Although this exploitation model does not result in direct economic benefit, it increases visibility and standing of UPC, which, in turn, we expect to produce economic benefits in the future.

In this line we also propose to exploit the deep understanding of the algorithms we developed and implemented to develop specialized, tailor-made solutions to problems coming both from the private and public sectors. We already followed this strategy in the past, e.g. through the development of computational models for superconductors for simulations required by the private sector (OXOLUTIA SL) or the development of FEMPAR-AM, a module for the simulation of additive manufacturing exploited by a private company (Stam) in a Cloudflow project experiment.

The UQ capabilities developed within FEMPAR for the ExaQUte project are and will be further exploited in an ongoing TecnioSpring + project (DaSim) to develop data-driven simulations for fluid flow in fractured media, as required by the private sector's (AMPHOS 21) interests in subsurface resource management. The DaSim project relies on developing iterative statistical methods building upon the methods used in this project, as well as the inclusion of data. These iterative statistical methods are a natural extension of the methods developed in the ExaQUte project, falling under the Bayesian and the machine learning paradigms. Like those algorithms developed in the ExaQUte project, those developed in the DaSim project are sufficiently general to be applicable for further exploitation in future projects.

\subsection{TUM exploitation plan}

The Chair of Structural Analysis led by Prof. Dr. -Ing. Kai-Uwe Bletzinger and the Chair of Numerical Mathematics led by Prof. Dr. Barbara Wohlmuth are both scientific departments at the Technical University of Munich (TUM) leading in the scientific fields of Numerical Methods in Engineering and Numerical Analysis by developing and testing sophisticated numerical algorithms and programs for tackling problems arising in the real engineering world. In particular, the Chair of Structural Analysis demonstrates expertise in the fields of Finite Element Methods (FEM) and Isogeometric Analysis (IGA) for Thin-Walled Structures among others, geometrically nonlinear analysis for Structural Mechanics and Structural Dynamics, Form-Finding of Membrane Structures, Structural and Multidisciplinary Optimization with Direct, Semi- or Analytical Sensitivity Analysis, Coupled Fluid-Structure Interaction, computational wind engineering etc. At the same time, the Chair of Numerical Mathematics also demonstrates expertise in the fields of FEM and IGA with a more particular focus on their numerical properties, Domain Decomposition Methods (DDMs), solutions to Partial Differential Equations (PDE's) on multiple domains, etc.

The expertise at the two chairs are brought together within the ExaQUte project in order to tackle problems arising in Wind Engineering and in particular the stochastic optimization of structures subject to wind loads. Monte Carlo (MC) Simulation and Multi-Level Monte Carlo (MLMC) method is accordingly selected as the method of choice as already existing deterministic optimization frameworks can be efficiently reused.

The following functionalities are going to be supported by TUM within the ExaQUte project:

- The computation of stochastic sensitivities for turbulent flows

- The framework for a stochastic gradient-based optimization method using Monte Carlo and MultiLevel Monte Carlo algorithms 
- Goal-oriented adaptive mesh refinement for high Reynolds number fluid flows

- The use of the exact geometry in terms of Computer-Aided Design (CAD) in terms of NonUniform Rational B-Splines (NURBS) for performing embedded Computational Fluid Dynamics (CFD) simulations for bluff bodies

- The use of the exact CAD geometry for performing stochastic optimization considering large shape updates

- Fast computation of synthetic turbulent wind fields

- Adaptive sampling strategies for stochastic optimization

- Fast assembly methods for IGA applications 